УДК: 005.32; 005.94; 005.95/.96

JEL: Z190; M140

Е. Ю. Благов ${ }^{1}$, Е. В. Гиленко ${ }^{1}$, А. С. Башлькова ${ }^{2}$

ДИФФЕРЕНЦИАЛ КУЛЬТУРНЫХ ИЗМЕРЕНИЙ КАК МЕТОД АНАЛИЗА ВНУТРИГРУППОВЫХ ПРОЦЕССОВ В КРОСС-КУЛЬТУРНЫХ КОМАНДАХ

${ }^{1}$ Санкт-Петербургский государственный университет,

Российская Федерация, 199034, Санкт-Петербург, Университетская наб., 7-9

${ }^{2}$ Международная Школа Бизнеса Хальт,

Кембридж, Массачусетс, США 02141, Education Street, 1

В статье представлен метод анализа влияния культурных факторов на внутригрупповые процессы в кросс-культурных командах, основанный на исследовании дифференциалов культурных измерений между их участниками. Предлагаемый метод ориентирован на нивелирование характерной для исследований в области кросс-культурного менеджмента методологической проблемы недостаточного внимания к внутригрупповому уровню анализа. Эмпирическая апробация метода производится на выборке кросскультурных студенческих команд, выполняющих групповые проектные работы.

Ключевые слова: кросс-культурный менеджмент, кросс-культурные команды, внутригрупповые процессы, модель GLOBE.

\title{
CROSS-CULTURAL DIMENSIONS DIFFERENTIAL AS A METHOD FOR ANALYZING IN-GROUP PROCESSES IN CROSS-CULTURAL TEAMS
}

\author{
E. Yu. Blagov ${ }^{1}$, E. V. Gilenko ${ }^{1}$, A.S. Bashlykova ${ }^{2}$
}

${ }^{1}$ St. Petersburg State University,

7-9, Universitetskaya nab., St. Petersburg, 199034, Russian Federation

${ }^{2}$ Hult International Business School, 1 Education Street, Cambridge, MA, 02141, USA

The purpose of the study represented in the article was to create and empirically test a method of analyzing in-group processes in cross-cultural teams, based on looking into the differentials between the highest and lowest values of cultural dimensions (e.g., power distance, uncertainty avoidance, etc.) between the group participants.

Empirical testing of the suggested method is done on the sample of cross-cultural student teams working on group projects while studying at GSOM SPbU Bachelor and Master educational programs. The differentials of such cultural dimensions as power distance, uncertainty avoidance, gender equality, institutional collectivism and in-group collectivism are analyzed as factors influencing the project participants' subjective perception of the team's efficiency and effectiveness of working on the project.

The results of the study revealed the statistically significant results consistent with the theoretical models and empirical research results present in contemporary cross-cultural

() Санкт-Петербургский государственный университет, 2016 
Дифференциал культурных измерений как метод анализа внутригрупповых процессов...

management literature, and also affording to look into the in-group mechanisms of how does the cross-cultural factors balance in the group does influence the parameters of the team work.

Thus, the suggested method demonstrates theoretical novelty and originality, as it is deepening existing methods of cross-cultural team research. The practical implications of the suggested method and the results of its empirical testing is also evident, allowing constructing teams for performing different project task types being based on differentials of cultural dimensions analyzed in this research.

However, the results of the research have certain limitations, based on the relatively small size of the research sample, consisting of 84 respondents, which is more or less suitable for the pilot study but is undoubtedly small for a full-fledged empirical research. Thus, although the suggested research method demonstrates to be valid and the results of its empirical testing are consistent with theory and results of acknowledged empirical studies, the empirical research component of the study needs to be repeated on the bigger samples. model.

Keywords: cross-cultural management, cross-cultural teams, in-group processes, GLOBE

\section{ВВЕДЕНИЕ}

Основным предметом исследования кросс-культурного менеджмента как отдельной области знаний является влияние культурных различий между человеческими общностями на управление организациями. Существенной методологической проблемой при этом выступает недостаточное внимание к тому, как изменения культурных факторов влияют на деятельность организации на уровне внутриорганизационных ${ }^{1}$ и внутригрупповых процессов, включая самостоятельные процессы в формальных и неформальных группах в составе организации. Как правило, организации в данной области исследований воспринимаются в качестве «черных ящиков», реагирующих на трансформацию внешних по отношению к ним факторов изменением результатов своей деятельности.

В настоящей статье разрабатывается и эмпирически апробируется метод анализа внутригрупповых процессов в кросс-культурных командах ${ }^{2}$, основанный на исследовании дифференциалов культурных измерений. Эмпирическая апробация метода представляет собой исследование влияния дифференциалов культурных измерений в кросс-культурных командах студентов, выполнявших работы группового проектного характера во время обучения на основных образовательных программах (ООП) бакалавриата и магистратуры в Институте «Высшая школа менеджмента» СПбГУ³, на воспринимаемую данными студентами успешность совместных проектов.

1 В дальнейшем в статье будет использоваться термин «внутригрупповые процессы», так как речь идет о процессах, которые могут происходить в любых группах из нескольких человек, необязательно совпадающих с организационными границами каких-либо организаций.

2 Термины «кросс-культурная группа» и «кросс-культурная команда» в данной статье используются как синонимы.

3 Решение опросить студентов именно данных программ было принято, помимо прочего, опираясь на метод «удобной выборки» (convenience sampling), т.е. подбора респондентов, получение ответов от которых представляет наименьшую сложность [Hall et al., 2012]. Однако, как показано далее, изучение этой выборки достаточно релевантно цели настоящей работы. 
Пилотное исследование продемонстрировало валидность предлагаемого метода. Полученные результаты соответствуют представленным в мировой литературе теоретическим моделям и выводам эмпирических работ в области анализа деятельности кросс-культурных групп, включая студенческие (см., напр.: [Bernardi, 2006; Ferdman, Sagiv, 2012; Ching et al., 2014; Griffith, Rubera, 2014; Ralston et al., 2014]). Вместе с тем ввиду малого размера эмпирической выборки требуется дальнейшая проверка на выборках более крупного размера.

Статья состоит из теоретической части, обосновывающей предлагаемый метод, и эмпирической части, представляющей дизайн пилотного исследования, проводимого для апробации метода, описание выборки исследования и анализ полученных результатов. В заключении делаются выводы о преимуществах и возможных направлениях дальнейшего развития метода.

\section{ТЕОРЕТИЧЕСКИЕ И МЕТОДОЛОГИЧЕСКИЕ ОСНОВЫ ИССЛЕДОВАНИЯ ВНУТРИГРУППОВЫХ ПРОЦЕССОВ В КРОСС-КУЛЬТУРНЫХ КОМАНДАХ}

Проблемы анализа внутригрупповых процессов в кросс-культурных командах. Основополагающие для дисциплины кросс-культурного менеджмента работы [Hall, 1959; Hofstede, 1980; Trompenaars, 1994] носят преимущественно описательный характер, выделяя по итогам интерпретации результатов эмпирических исследований культурные измерения (индивидуализм/коллективизм, избегание неопределенности и т.п.), способные влиять на деятельность организаций.

С дальнейшим развитием дисциплины кросс-культурного менеджмента стали появляться и эмпирические исследования влияния различий в культурных измерениях на деятельность организаций. Однако, при безусловной теоретической, методологической и прикладной ценности имеющейся эмпирической литературы в области кросс-культурного менеджмента, значительной ее части присущ методологический недостаток. Он заключается в том, что при исследовании влияния культурных факторов на деятельность организации последняя зачастую рассматривается в качестве «черного ящика», реагирующего на влияние внешних факторов изменением параметров собственной деятельности или ее результатов (например, уровня выручки, издержек и прибыли коммерческой фирмы). При этом незначительное внимание уделяется тем внутригрупповым процессам, которые происходят под воздействием внешних факторов и служат причиной изменения результатов деятельности организации [Ferdman, Sagiv, 2012; House et al., 2004].

Как правило, в качестве внешних действующих факторов (независимых переменных) в исследованиях в области кросс-культурного менеджмента выступают изменения уровня культурных измерений отдельных сотрудников либо средних уровней культурных измерений организаций в целом.

Представляется, что показатели средних уровней культурных измерений в организациях слабо отражают внутригрупповые процессы, так как одни и те же 
Дифференциал культурных измерений как метод анализа внутригрупповых процессов...

средние значения могут быть получены в группах с разным разбросом значений вокруг этой средней и, соответственно, разной степенью культурной однородности организации (безусловно, сильно влияющей на взаимоотношения между сотрудниками организации и, соответственно, на происходящие в организации процессы [Ferdman, Sagiv, 2012; Tsai et al., 2012]). Тем более сложно вести речь о том, как на эти процессы влияют изменения уровней культурных измерений отдельных сотрудников [Connaughton, Shuffler, 2007].

Для исследования внутригруппового взаимодействия носителей различных уровней культурных измерений целесообразно использовать разности значений данных уровней. При наличии в группе более двух участников разность между наиболее близкими уровнями каких-либо культурных измерений может служить мерой качества коммуникации в группе [Spony, 2003].

Не менее интересным представляется и изучение дифференциала культурных измерений, т. е. разности наибольшего и наименьшего значений какого-либо культурного измерения в группе 4 . Дифференциал культурных измерений может продемонстрировать «напряженность связей» между носителями различных уровней культурных измерений и потенциал взаимодополнения качеств этих носителей (либо, наоборот, конфликта между ними). В имеющихся исследованиях данный вид разности значений для анализа внутригрупповых процессов в кросскультурных группах не использовался. В настоящей работе предпринята попытка разработать и эмпирически апробировать метод анализа таких процессов, основанный на данном параметре.

Развитие ситуаций взаимодополнения либо конфликта выступает существенным элементом внутригрупповых процессов, которые влияют на результаты деятельности группы [Sheikh, Newman, Al Azzeh, 2013]. Соответственно исследование дифференциалов культурных измерений может лежать в основе метода, позволяющего анализировать подобные процессы, и, таким образом, подробно изучать механизмы влияния изменений уровней культурных измерений участников группы на результаты ее деятельности.

Кросс-культурные команды студентов как объект исследования внутригрупповых процессов. Важной проблемой исследований в области кросскультурного менеджмента является относительно узкая применимость конкретных методик и конкретных результатов, так как в зависимости от контекста изменения уровней культурных измерений могут оказывать на организации совершенно разное влияние. Тем более значимым этот вопрос является для настоящей статьи, посвященной разработке и эмпирической апробации аналитического

\footnotetext{
4 Вместо термина «разность наибольшего и наименьшего значений культурного измерения» мы предлагаем использовать термин дифференциал культурных измерений. Это объясняется тем, что слово «дифференциал» применяется в названиях методически схожих исследовательских подходов как в социальных науках в целом («семантический дифференциал» (semantic differential, [Osgood, 1952]), так и в кросс-культурном менеджменте в частности («культурно-ценностный дифференциал» [Солдатова, 1998]).
} 
метода, результаты применения которого в заданном контексте могут оказаться совершенно нерелевантными в других условиях.

В качестве объекта пилотной эмпирической апробации разрабатываемого метода выбраны кросс-культурные команды студентов, обучающихся на образовательных программах в области менеджмента, а именно: ООП бакалавриата и магистратуры по направлениям «Менеджмент» и «Государственное и муниципальное управление» Института «Высшая школа менеджмента» СПбГУ. Данный выбор обусловлен тем, что, согласно Федеральным государственным образовательным стандартам по уровням образования «бакалавриат» и «магистратура» по данным направлениям, помимо компетенций, связанных с узкофункциональными областями менеджмента (финансовый менеджмент, маркетинг, логистика и т. п.), в ходе получения образования формируются также и компетенции общеуправленческого характера, применимые к управлению любой организацией 5 . Соответственно «удобную» выборку студентов можно рассматривать как аналог выборок реальных менеджеров, занимающихся различными видами профессиональной управленческой деятельности.

Кросс-культурная среда, охватывающая студентов из различных стран, может формироваться в рамках изучения любых дисциплин, предлагаемых на ООП бакалавриата и магистратуры по направлениям «Менеджмент» и «Государственное и муниципальное управление». Однако кросс-культурные группы студентов, выполняющие специфические задачи, представлены не во всех видах учебной деятельности. Например, текущая или промежуточная аттестация проводится в индивидуальном порядке. Факторы, связанные с культурными переменными в группе, в таком случае влияют на индивидуальные результаты работы каждого из ее участников, однако эти результаты не являются итогом целенаправленной деятельности всей группы.

В настоящем исследовании нас интересует именно групповая деятельность, подразумевающая результаты целенаправленных совместных действий участников группы (вне зависимости от того, одинаковы они для разных ее участников или нет). Соответственно, среди различных видов учебной деятельности выбраны групповые проекты, выполняемые кросс-культурными группами студентов.

Зависимыми переменными при исследовании факторов, влияющих на успешность выполнения групповых проектов, могут быть как внешние (оценка внешними лицами), так и внутренние (оценка ее участниками) критерии оценки работы группы над проектом.

5 Например, Приказ министра образования и науки Российской Федерации от 20 мая 2010 г. № 544 «О внесении изменений в федеральные государственные образовательные стандарты высшего профессионального образования» [О внесении изменений..., 2010] подразумевает для бакалавриата по направлению «Менеджмент» такие виды профессиональной деятельности, как организационно-управленческая, предпринимательская и информационно-аналитическая, а для магистратуры - организационно-управленческая, аналитическая, научно-исследовательская, педагогическая деятельность. 
Дифференциал культурных измерений как метод анализа внутригрупповых процессов...

Наиболее очевидным внешним критерием успешности выполнения проектов являются оценки, полученные участниками групп. Преимущество данного критерия в том, что оценки имеют количественное выражение. Однако объективность этого критерия ограничивается рядом факторов.

Во-первых, различие в получаемых студентами оценках может являться следствием субъективного восприятия преподавателями каких-либо иных факторов, не имеющих отношения к культурным параметрам группы. Во-вторых, использование итоговых оценок для формирования зависимых переменных выступает примером восприятия группы в качестве «черного ящика», в то время как разрабатываемый нами метод ориентирован на рассмотрение процессов, происходящих внутри него.

Внутренние критерии оценки работы группы над проектом более релевантны для формирования зависимых переменных, так как участники группы, как правило, имеют больше информации о событиях, чем внешние лица [Singh, Joshi, 2015]. Какого рода внутренние критерии могут быть наиболее релевантными?

Проекты, выполняемые группами студентов, предусматриваются рабочими программами большинства дисциплин, освоение которых осуществляется в рамках компетентностно-ориентированных учебных планов образовательных программ. Соответственно, данные проекты могут быть ориентированы на формирование самых различных компетенций как общеуправленческого, так и узкопрофильного характера. Поэтому возникает необходимость в использовании максимально общих критериев оценки студентами своей работы над проектами.

Разработка субъективных критериев оценки студентами своей работы, включая выполнение групповых проектов, является достаточно актуальным направлением исследований в области управления образованием, в том числе и бизнесобразованием (в частности, речь об этом идет в: [Samkin, Francis, 2008; Mansour, 2015; Singh, Joshi, 2015]).

Применительно к настоящему исследованию речь может идти о следующих критериях. В первую очередь осуществление групповых проектов в рамках рабочей программы любой дисциплины подразумевает не просто выполнение предлагаемых преподавателем инструкций, но и проявление творческого начала группы, выражающегося в генерации собственных идей [Samkin, Francis, 2008]. Соответственно среди субъективных критериев внутренней оценки успешности работы над проектом особую значимость имеет успешность группы в генерации новых идей.

Помимо генерации идей, ключевыми критериями успеха любого проекта являются критерии, связанные с процессами управления проектом - планированием, организацией, мотивацией участников проекта и контролем их деятельности [Besteiro, de Souza Pinto, Novaski, 2015]. В работах [Abbondante et al., 2014; Sormunen et al., 2014]. К важным критериям отнесены также качество (четкость) делегирования задач в рамках выполнения проекта и его результативность, понимаемая как своевременность выполнения поставленных задач.

Наконец, в исследованиях [Lehmann, Heagy, Wilson, 2011; Gonzalez-Padron, Ferguson, 2015] указывается и на значимость критериев, связанных с развитием 
информационно-аналитических навыков студентов в ходе выполняемых проектов; в первую очередь в качестве субъективного критерия, который может отражать реальное развитие подобных навыков, предлагается качество анализа используемой инбормации.

В настоящей статье в качестве критериев успешности организационной работы над проектами предлагаются четкость делегирования и своевременность выполнения задач в процессе работы над проектом.

Культурные измерения как нормативные ценности и социальные практики. Для формирования независимых переменных исследования используется ряд культурных измерений модели, разработанной в рамках исследовательского проекта Global Leadership and Organizational Behavior Effectiveness Research Program (GLOBE), описываемого в [House et al., 2004]. Эта модель была выбрана в связи с тем, что культурные измерения анализируются в ней с двух точек зрения: как нормативные ценности (social values; для краткости мы применяем формулировку «культурные измерения $S V »)$ и как социальные практики (social practices; «культурные измерения $S P »)$. Эмпирические данные по значениям включенных в модель культурных измерений в разных странах также собраны и по нормативным ценностям, и по социальным практикам.

Данные по показателям культурных измерений для различных стран формировались путем анкетирования представителей национальной деловой среды каждой страны ${ }^{6}$ с помощью набора шкал Лайкерта со значениями от 1 до 7. Соответственно, усредненные значения показателей какого-либо культурного измерения по странам - это дискретная переменная со значениями от 1,00 до $7,00^{7}$.

Среди культурных измерений, входящих в модель GLOBE, нами были выделены те, которые непосредственно развивают «классические» измерения, предложенные в [Hofstede, 1980; Trompenaars, 1994]. Таким образом, сравнимость результатов нашего исследования с методологией и выводами большинства научных работ по кросс-культурному менеджменту повышается.

К указанным измерениям можно отнести следующие:

- дистанция власти (power distance) - степень толерантности общества к неравномерному распределению власти в социальных институтах и организациях;

- избегание неопределенности (uncertainty avoidance) - степень стремления к избеганию ситуаций, могущих создавать воспринимаемую угрозу по причинам неопределенности или двусмысленности;

- гендерное равенство (gender equality) - степень толерантности общества к равенству социальных ролей женского и мужского гендера;

6 Согласно данным, приводимым в [House et al., 2004, p. 96], среднее количество респондентов на страну составило 251 чел.

7 В данной статье, как и в работе [House et al., 2004], используются значения с двумя знаками после запятой. 
Дифференциал культурных измерений как метод анализа внутригрупповых процессов...

- институциональный коллективизм (institutional collectivism) - степень лояльности индивидов к формальным социальным институтам, выражающаяся в склонности ориентироваться на помощь формальных институтов и содействовать их функционированию;

- внутригрупповой коллективизм (in-group collectivism) - степень лояльности индивидов к малым, в том числе и неформальным, группам.

В соответствии с моделью GLOBE каждое из данных измерений рассматривается нами в двух аспектах как нормативная ценность и как социальная практика. Поэтому для каждого из них формируется по две независимые переменные.

Итак, в эмпирическом исследовании, предназначенном для апробации предлагаемого метода, используются следующие наборы переменных.

Четыре типа зависимых переменных:

1) успешность группы в генерации новых идей;

2) четкость делегирования задач в рамках выполнения проекта;

3) своевременность выполнения задач в ходе работы над проектом;

4) качество анализа используемой информации.

Для каждого из этих типов, в соответствии с разработанной для проведения опроса анкетой введены три зависимые переменные (далее - $d v$ (dependent variables)). Всего рассматривается 12 зависимых переменных.

Десять независимых переменных:

1) дифференциал дистанции власти как нормативной ценности (PDISV);

2) дифференциал дистанции власти как социальной практики (PDISP);

3) дифференциал избегания неопределенности как нормативной ценности (UAISV);

4) дифференциал избегания неопределенности как социальной практики (UAISP);

5) дифференциал гендерного равенства как нормативной ценности (GendEqSV);

6) дифференциал гендерного равенства как социальной практики (GendEqSP);

7) дифференциал институционального коллективизма как нормативной ценности (InstColsV);

8) дифференциал институционального коллективизма как социальной практики (InstColSP);

9) дифференциал внутригруппового коллективизма как нормативной ценности (InGroupColSV);

10) дифференциал внутригруппового коллективизма как социальной практики (InGroupColSP). 


\section{ЭМПИРИЧЕСКАЯ АПРОБАЦИЯ \\ МЕТОДА ИССЛЕДОВАНИЯ \\ ДИФФЕРЕНЦИАЛА КУЛЬТУРНЫХ ИЗМЕРЕНИЙ \\ В КРОСС-КУЛЬТУРНЫХ КОМАНДАХ}

Анкета и выборка исследования. Эмпирическое исследование проводилось с помощью эконометрического анализа результатов анкетирования студентов бакалавриата и магистратуры Института «Высшая школа менеджмента» СПбГУ, состоявшегося в апреле - мае 2015 г.

На программах бакалавриата опрошивались студенты 4-го (выпускного) курса, имевшие предположительно максимальный опыт работы в кросс-культурных командах, в составе которых были иностранные студенты, проходившие включенное обучение в ВШМ. Студентам было разослано 190 анкет.

На программах магистратуры анкеты адресовались студентам обоих курсов (всего 207 человек) ввиду более высокой степени интернационализации программ, предполагающих возможность обучения студентов по обмену на любой из дисциплин. Анкетирование проводилось в период весеннего семестра, поэтому опыт работы в кросс-культурных студенческих группах к моменту анкетирования могли получить и студенты 1-го курса.

Таким образом, всего было разослано 397 анкет. Анкетирование осуществлялось с помощью онлайн-анкеты, размещенной на электронном pecypce Qualtrics. com. Анкета состояла из 16 вопросов.

Первые четыре вопроса анкеты являлись вступительными. Два первых касались пола и возраста респондентов. Третий вопрос устанавливал, принимал ли респондент участие в выполнении кросс-культурных проектов во время обучения в ВШМ. В случае утвердительного ответа опрос продолжался; респондента просили описать кросс-культурный проект, наиболее запомнившийся из выполнявшихся во время обучения. В четвертом вопросе респонденты перечисляли национальный состав участников группы, выполнявшей данный проект.

Зависимые переменные исследования (всего 12) соответствуют вопросам 5.1-8.3 анкеты. Как отмечалось, они базируются на четырех субъективно воспринимаемых участниками проектов критериях их успешности. Для более точного оценивания каждого из этих критериев используется три вопроса со шкалой ответа на базе шкалы Лайкерта с десятью возможными вариантами ответа: от 1 («Полностью не согласен с приведенным утверждением») до 10 («Полностью согласен с приведенным утверждением»).

Два вопроса из каждой тройки дублируют друг друга по смыслу, чтобы путем создания избыточности вопросов повысить качество получаемых ответов. Примером подобного дублирования могут служить вопросы 6.1 («Во время выполнения проекта каждый участник четко знал, какие конкретно задачи он выполняет») и 6.3 («Задание каждого участника было четко определено внутри группы»). 
Дифференциал культурных измерений как метод анализа внутригрупповых процессов...

Формулировки вопросов 5.1-8.3 анкеты имеют следующий вид.

5. Успешность группь в генерации новых идей:

5.1) генерация идей в группе не вызывала затруднений;

5.2) участники всегда были готовы выслушать друг друга;

5.3) члены группы в ходе работы часто подавали новые интересные идеи.

6. Четкость делегирования задач в рамках выполнения проекта:

6.1) во время выполнения проекта каждый участник четко знал, какие конкретно задачи он выполняет;

6.2) во время выполнения проекта каждый участник четко знал, над какими задачами работают остальные члены команды;

6.3) задание каждого участника было четко определено внутри группы.

7. Своевременность выполнения задач в рамках работы над проектом:

7.1) во время работы над проектом каждый участник выполнял свои задачи вовремя;

7.2) за время выполнения проекта группе удавалось придерживаться поставленных сроков;

7.3) работа была завершена и сдана в срок.

8. Качество анализа используемой информаиии:

8.1) для выполнения проекта группа старалась использовать всю доступную информацию;

8.2) необходимая для работы над проектом информация анализировалась максимально глубоко;

8.3) участники группы искали дополнительные источники информации (часто ходили в библиотеку, искали редкие издания, консультировались с преподавателями/представителями фирмы).

Для увеличения достоверности ответов порядок вопросов в анкете был изменен с тем, чтобы соседние вопросы не относились к зависимым переменным одного типа.

Респонденты отвечали и на вопрос о национальном составе описываемых ими кросс-культурных групп, поэтому для каждой из них вычислялся максимальный дифференциал (разница между наблюдаемым в группе наибольшим и наименьшим значениями) всех культурных измерений и как нормативной ценности $(S V)$, и как социальной практики $(S P)$.

Для нахождения этих дифференциалов делалось допущение, что каждый из участников описываемых групп является типичным представителем своей национальной управленческой культуры. Данные по значениям культурных измерений $S V$ и $S P$ для различных национальных культур, на базе которых для каждой группы вычислялись дифференциалы, брались из результатов исследований в рамках проекта GLOBE, представленных в [House et al., 2004]. Указанное допущение может исказить истинные величины культурных измерений в группе, так как в рамках сколь угодно однородного по этническим, социальным и иным 
признакам коллектива могут наблюдаться различные значения культурных измерений [Connaughton, Shuffler, 2007]. Однако целью исследования являлось не формулирование конкретных управленческих рекомендаций, а разработка и апробация исследовательского метода, поэтому данное допущение все же не представляется чрезмерно ограничивающим.

Из 397 респондентов на вопросы ответили 110 (процент отклика - 27,7\%), при этом на все вопросы - лишь 84 человека. Их ответы и использовались в настоящем анализе. Соответственно итоговый процент отклика составил $21,2 \%$.

Из предоставленных 84 ответов 21 ответ (25\%) описывает группы, состоящие из граждан двух стран; 32 ответа $(38,1 \%)$ - трех стран, и 31 ответ $(36,9 \%)$ - группы из граждан четырех и более стран.

Распределение 84 респондентов по гендерному признаку оказалось неравномерным (29 мужчин и 55 женщин). В этой связи была проведена проверка «равномерности» ответов респондентов - не завышали или не занижали ли в среднем мужчины либо женщины оценки своих ответов. Данная процедура была осуществлена с помощью стандартного теста Стьюдента ( $t$-теста) на равенство истинных средних двух выборок (нулевая гипотеза теста состоит в том, что истинные средние двух выборок совпадают). Результаты этого теста для всех рассматриваемых зависимых переменных представлены в табл. 1. Как видно, p-значения при тестировании всех зависимых переменных оказались выше традиционного уровня

Таблица 1 . Результаты $\boldsymbol{t}$-теста на равенство средних значений зависимых переменных для двух гендерных групп

\begin{tabular}{|c|c|c|}
\hline $\begin{array}{c}\text { Зависимая } \\
\text { переменная }\end{array}$ & $t$-статистика & $p$-значение \\
\hline$d v 51$ & 0,04 & 0,9703 \\
\hline$d v 52$ & 0,71 & 0,4829 \\
\hline$d v 53$ & $-1,04$ & 0,3013 \\
\hline$d v 61$ & 0,42 & 0,6729 \\
\hline$d v 62$ & 1,05 & 0,2968 \\
\hline$d v 63$ & 0,81 & 0,4204 \\
\hline$d v 71$ & $-0,01$ & 0,9912 \\
\hline$d v 72$ & $-1,02$ & 0,3113 \\
\hline$d v 73$ & $-0,58$ & 0,5646 \\
\hline$d v 81$ & $-0,19$ & 0,8522 \\
\hline$d v 82$ & 0,11 & 0,9143 \\
\hline$d v 83$ & $-0,10$ & 0,9185 \\
\hline
\end{tabular}

* Примечан и е: нумерация зависимых переменных основана на нумерации вопросов анкеты в соответствующих блоках. Так, переменные $d v 51-d v 53$ соответствуют ответам на вопросы 5.1-5.3, переменные $d v 61-d v 63$ - на вопросы 6.1-6.3, раскрывающие критерий «Четкость делегирования задач в рамках выполнения проекта», и т. д. 
Дифференциал культурных измерений как метод анализа внутригрупповых процессов...

значимости 0,05. Это означает, что для этих переменных гипотеза о равенстве их средних значений для двух гендерных групп не отвергается, а соответственно распределение оценок респондентов по гендерному признаку представлено равномерно.

Методология количественного анализа результатов эмпирического исследования. Обработка результирующего набора данных проводилась в два этапа. На первом этапе применялась процедура иерархического кластерного анализа, а на втором - в сформированных кластерах оценивались соответствующие линейные регрессионные модели для выбранных зависимых и независимых переменных.

Поскольку изначально полученный набор данных был довольно неоднородным по своей структуре, в целях выделения более однородных кластеров наблюдений применялась процедура иерархического кластерного анализа к обоим наборам независимых переменных (культурным измерениям как нормативным ценностям и как социальным практикам). В результате было выделено по четыре кластера наблюдений.

Средние значения дифференциалов культурных измерений в соответствующих кластерах приведены в табл. 2. Последующий линейный регрессионный анализ проводился в рамках выделенных кластеров.

Таблица 2. Средние значения дифференциалов культурных измерений типа «нормативные ценности» $(S V)$ и «социальные практики» $(S P)$ по кластерам

\begin{tabular}{|c|c|c|c|c|c|}
\hline Кластер & GendEqSV & InstColSV & InGroupColSV & PDISV & UAISV \\
\hline 1 & 0,615 & 0,557 & 0,592 & 0,312 & 0,944 \\
\hline 2 & 0,962 & 0,667 & 1,431 & 0,496 & 2,271 \\
\hline 3 & 0,662 & 0,622 & 1,332 & 0,489 & 1,376 \\
\hline 4 & 0,989 & 1,174 & 1,878 & 0,873 & 1,991 \\
\hline Кластер & GendEqSP & InstColSP & InGroupColSP & PDISP & UAISP \\
\hline 1 & 0,805 & 1,451 & 0,394 & 0,451 & 0,753 \\
\hline 2 & 0,811 & 1,043 & 0,575 & 0,371 & 1,559 \\
\hline 3 & 0,626 & 0,574 & 0,379 & 0,334 & 0,824 \\
\hline 4 & 1,351 & 1,144 & 0,942 & 0,929 & 2,043 \\
\hline
\end{tabular}

Основной метод количественного анализа, используемый в рамках настоящего исследования, - линейный регрессионный анализ. Он проводился для каждой из 12 зависимых переменных в каждом из 4 кластеров для каждого из двух наборов ( $S V$ и $S P)$ объясняющих переменных (всего 96 регрессий). Типовое регрессионное уравнение имеет следующую спецификацию:

$$
\begin{gathered}
D V_{i}=\beta_{0}+\beta_{1} \cdot d \text { GendEq }_{i}+\beta_{2} \cdot d \operatorname{InstCol}_{i}+\beta_{3} \cdot d \operatorname{InGroupCol} \text { Cl }_{i}+ \\
+\beta_{4} \cdot d P D I_{i}+\beta_{5} \cdot d U A I_{i}+\varepsilon_{i} .
\end{gathered}
$$


Показатель $D V$ обозначает любую (отсюда - написание прописными буквами) из 12 зависимых переменных $(d v)$ исследования (соответствующих ответам на каждый из трех вопросов блоков, относящихся к четырем субъективно воспринимаемым критериям успешности работы кросс-культурной группы). Независимые переменные, в свою очередь, представляют собой максимальные дифференциалы (как $S V$, так и $S P$ ) используемых в исследовании культурных измерений: гендерное равенство (GendEq), институциональный коллективизм (InstCol), внутригрупповой коллективизм (InGroupCol), дистанция власти (PDI) и избегание неопределенности (UAI).

После оценивания каждого из уравнений регрессии были проведены необходимые диагностические тесты, в частности - тест на гетероскедастичность остатков регрессионного уравнения, который в подавляющем большинстве случаев дал положительный результат. Соответственно, оценивание регрессионных уравнений проводилось с использованием процедуры робастного расчета стандартных ошибок.

\section{РЕЗУЛЬТАТЫ ЛИНЕЙНОГО РЕГРЕССИОННОГО АНАЛИЗА}

Кластерный анализ наборов независимых переменных $S V$ и $S P$ привел к выделению четырех самостоятельных кластеров. Поэтому необходимо рассмотреть результаты анализа для данных наборов переменных по отдельности.

В табл. 3 представлены результаты линейного регрессионного анализа для набора переменных $S V$.

Прежде чем последовательно рассмотреть результаты для различных независимых переменных дифференциалов культурных измерений $S V$, следует отметить общую для них тенденцию, которая, как представляется, играет значимую роль для исследования внутригрупповых процессов в кросс-культурных командах.

В соответствии с трактовкой различий между культурными измерениями как нормативными ценностями и как социальными практиками [House et al., 2004] культурные измерения в качестве нормативных ценностей можно трактовать как восприятие недостающих качеств национальной культуры. Соответственно можно предположить, что увеличение дифференциалов культурных измерений набоpa $S V$ будет приводить к эффекту наложения и взаимного усиления качеств, недостающих или проблемных для соответствующих национальных деловых культур.

Однако если бы это касалось всех рассматриваемых культурных измерений, среди статистически значимых коэффициентов при дифференциалах $S V$ преобладали бы коэффициенты с отрицательным знаком, показывающие ухудшение качества работы кросс-культурных команд с увеличением дифференциала соответствующих культурных измерений.

Как показано в табл. 3, подобное рассуждение оправдывается лишь для независимой переменной In GroupColsV (1 положительный и 25 отрицательных статистически значимых коэффициентов при выбранном уровне значимости), в то 
Дифференциал культурных измерений как метод анализа внутригрупповых процессов...

Таблица 3. Результаты линейного регрессионного анализа для набора переменных $S V$

\begin{tabular}{|c|c|c|c|c|c|c|c|c|c|c|c|c|c|}
\hline \multirow[t]{2}{*}{ Переменные } & \multirow{2}{*}{$\bigvee_{\text {Кластер }}^{\text {Вопрос }}$} & \multicolumn{3}{|c|}{$\begin{array}{c}\text { Успешность } \\
\text { группы в гене- } \\
\text { рации новых } \\
\text { идей }\end{array}$} & \multicolumn{3}{|c|}{$\begin{array}{c}\text { Четкость } \\
\text { делегирования } \\
\text { задач в рамках } \\
\text { выполнения } \\
\text { проекта }\end{array}$} & \multicolumn{3}{|c|}{$\begin{array}{c}\text { Своевремен- } \\
\text { ность выполне- } \\
\text { ния задач в ходе } \\
\text { работы над } \\
\text { проектом }\end{array}$} & \multicolumn{3}{|c|}{$\begin{array}{c}\text { Качество } \\
\text { анализа } \\
\text { используемой } \\
\text { информации }\end{array}$} \\
\hline & & 51 & 52 & 53 & 61 & 62 & 63 & 71 & 72 & 73 & 81 & 82 & 83 \\
\hline \multirow[t]{4}{*}{ PDISV } & 1 & + & - & - & - & - & - & - & - & - & - & - & - \\
\hline & 2 & - & + & + & + & + & - & + & + & + & + & + & + \\
\hline & 3 & + & + & + & + & + & + & + & + & - & + & + & + \\
\hline & 4 & - & + & + & - & - & + & - & + & - & - & - & + \\
\hline \multirow[t]{4}{*}{ UAISV } & 1 & - & - & - & - & + & - & - & + & - & - & - & - \\
\hline & 2 & + & + & + & + & + & + & + & - & - & - & + & - \\
\hline & 3 & + & + & + & + & + & + & + & + & + & + & + & + \\
\hline & 4 & + & + & + & - & - & + & - & - & + & - & - & + \\
\hline \multirow[t]{4}{*}{ GendEqSV } & 1 & - & + & - & - & - & - & - & - & - & - & - & + \\
\hline & 2 & + & + & + & - & + & - & + & + & + & - & - & + \\
\hline & 3 & + & + & + & - & - & + & - & - & + & + & + & - \\
\hline & 4 & - & + & - & + & - & - & + & + & - & - & - & + \\
\hline \multirow[t]{4}{*}{ InstColSV } & 1 & + & - & + & + & + & - & - & + & + & - & - & + \\
\hline & 2 & - & - & - & + & - & + & + & - & - & - & - & - \\
\hline & 3 & - & - & - & + & + & + & + & + & - & + & + & + \\
\hline & 4 & - & + & - & + & + & + & + & + & - & + & + & + \\
\hline \multirow[t]{4}{*}{ InGroupColsV } & 1 & - & - & - & - & - & - & - & - & + & - & - & - \\
\hline & 2 & - & - & - & - & - & - & - & - & - & - & - & - \\
\hline & 3 & - & - & - & - & - & - & - & - & - & - & - & - \\
\hline & 4 & + & - & + & + & + & - & + & - & + & - & + & - \\
\hline
\end{tabular}

П р и м е ч а н и е: серым и светло-серым цветами соответственно отмечены обладающие положительным и отрицательным знаком коэффициенты, статистически значимые на уровне значимости 0,2. Стандартные ошибки коэффициентов вычислялись с учетом поправки Уайта на гетероскедастичность. Полужирным выделены блоки коэффициентов, статистически значимых для всех трех вопросов, отражающих какую-либо из зависимых переменных для какого-либо кластера и, таким образом, показывающих для конкретного кластера статистически значимую на принятом уровне значимости связь между соответствующими зависимой и независимой переменными.

время как для остальных независимых переменных блока SV распределение знаков выглядит иначе.

Рассмотрим результаты для различных независимых переменных.

Дифференциал дистанции власти (PDISV). В коэффициентах при независимой переменной данного дифференциала наиболее заметны статистически значимые коэффициенты для всех трех вопросов зависимой переменной «Четкость делегирования задач...» в кластерах 1 и 3. При этом в кластере 1 они имеют отрицательный знак (как и отдельные коэффициенты при вопросах зависимых 
переменных «Успешность группы в генерации новых идей» и «Своевременность выполнения задач...»). В кластере 3 все статистически значимые коэффициенты при вопросах этой зависимой переменной имеют положительный знак, как и отдельные коэффициенты для вопросов зависимых переменных «Своевременность выполнения задач...» и «Качество анализа используемой информации».

Таким образом, можно констатировать наличие тенденции к увеличению количества положительных значимых коэффициентов с увеличением среднего дифференциала по кластерам. Однако в кластере 4 можно наблюдать по одному отрицательному значимому коэффициенту при вопросах зависимых переменных «Четкость делегирования задач...» и «Своевременность выполнения задач...».

Эти результаты позволяют заключить, что для кластера с минимальным уровнем среднего дифференциала $S V$ увеличение $P D I S V$ статистически значимо отрицательно влияет на субъективно воспринимаемую участниками группы четкость делегирования задач в группе. В то же время для кластера 3 его увеличение оказывает статистически значимое положительное влияние на четкость делегирования задач.

Следовательно, увеличение PDISV положительно влияет на успешность работы кросс-культурной команды при умеренном уровне культурного разнообразия команды в целом.

Дифференциал избегания неопределенности (UAISV). Все статистически значимые коэффициенты при независимой переменной UAISV в кластерах 2 и 3 являются положительными, а в кластерах 1 и 4 - как положительными, так и отрицательными.

Иными словами, увеличение UAISV для команд с умеренным уровнем культурного разнообразия в целом положительно влияет на воспринимаемую членами групп успешность их работы, в то время как для максимально или минимально культурно однородных команд влияние увеличения данного дифференциала может быть не только положительным, но и отрицательным.

Как объяснить подобный результат? Согласно [House et al., 2004], среди исследуемых в проекте GLOBE культурных измерений для избегания неопределенности характерны наиболее сильные негативные корреляции между значениями в разных странах культурного измерения как нормативной ценности $(S V)$ и как социальной практики (SP). Таким образом, избегание неопределенности как нормативная ценность в большей степени, чем другие культурные измерения $S V$ в модели GLOBE, является восприятием недостающих черт национальной культуры.

Можно предположить, что увеличение дифференциала воспринимаемых недостающих черт культуры будет стимулировать членов групп к поиску и реализации взаимодополняющих характеристик. Различие знаков статистически значимых коэффициентов в разных кластерах при этом можно трактовать следующим образом. В соответствии с [Griffith, Rubera, 2014] поиск взаимодополняющих высоких и низких уровней избегания неопределенности обычно бывает успешен, способствуя нахождению целей и постановке задач членами группы 
Дифференциал культурных измерений как метод анализа внутригрупповых процессов...

с относительно низким уровнем избегания неопределенности и исполнению поставленных задач - членами группы с относительно высоким уровнем. Однако для культурно однородной группы увеличение UAISV может не привести к нужному результату ввиду недостаточных различий между участниками группы для достижения работающего взаимодополнения [Ferdman, Sagiv, 2012]. Напротив, в группах с высоким уровнем культурного разнообразия поиск и использование взаимодополняющих культурных черт могут осложняться затруднениями во взаимопонимании между участниками [Tsai et al., 2012].

Вместе с тем, как показало настоящее исследование, для независимой переменной UAISV ни в одном из кластеров не обнаружено трех статистически значимых коэффициентов для всех вопросов, отражающих какую-либо из зависимых переменных. Соответственно, высказанные предположения требуют проверки на более многочисленной выборке респондентов.

Дифференциал гендерного равенства (GendEqSV). Знаки коэффициентов независимой переменной GendEqSV могут трактоваться следующим образом. Наиболее интересным представляется наличие статистически значимых отрицательных коэффициентов при отдельных вопросах всех зависимых переменных в кластерах 1 и 4, а также отсутствие статистически значимых коэффициентов с аналогичным знаком в кластерах 2 и 3. Статистически значимые коэффициенты с положительным знаком (за одним исключением из четырех - в кластере 4) находятся именно в кластере 2.

Как отмечалось, увеличение дифференциалов $S V$ потенциально может создать в группе конфликтогенную ситуацию, так как культурные измерения $S V$ выражают воспринимаемый недостаток эффективных черт национальной культуры. Соответственно, наличие статистически значимых отрицательных коэффициентов при переменной GendEqSV в кластере 1 можно объяснить как проявление негативного влияния увеличения дифференциала данного измерения при прочих равных в группах, в целом относительно культурно однородных.

В более культурно разнородных группах, обладающих более высоким средним дифференциалом $S V$, негативное влияние увеличения переменной GendEqSV может нивелироваться нахождением и реализацией взаимодополняющих черт [Knights, Tullberg, 2012]. Применительно к кластеру 4 с наиболее высоким уровнем культурного разнообразия увеличение дифференциала GendEqSV может приводить к возникновению чрезмерно конфликтогенной обстановки, способной создавать конфликты конструктивного характера лишь в редких случаяx [Knights, Tullberg, 2012]. Так, например, для настоящей выборки при переменной GendEqSV в данном кластере есть всего один статистически значимый положительный коэффициент.

Дифференциал институционального коллективизма (InstColSV). Независимая переменная InstColSV обращает на себя внимание рядом результатов. Всего два из 14 статистически значимых коэффициентов при данной переменной имеют отрицательный знак. К этим коэффициентам относятся коэффициент в первом 
вопросе зависимой переменной «Качество анализа используемой информации» в кластере 1 и коэффициент в третьем вопросе зависимой переменной «Своевременность выполнения задач...» в кластере 4. Остальные статистически значимые коэффициенты в кластерах 1 и 4, а также единственный статистически значимый коэффициент в кластере 3 являются положительными. В кластере 2, в свою очередь, статистически значимые коэффициенты отсутствуют, что позволяет сделать предположение о наименьшем влиянии увеличения InstColsV на успешность работы групп с уровнем культурного разнообразия по культурным измерениям $S V$, примерно соответствующим уровню кластера 2.

Наличие отрицательных статистически значимых коэффициентов в кластеpax 1 и 4 можно объяснить с помощью высказанного предположения о том, что увеличение дифференциалов культурных измерений $S V$ может негативно влиять на деятельность групп с чрезмерно низкими или высокими уровнями культурного разнообразия ввиду коммуникационных проблем, потенциально могущих служить источником конфликтов.

Однако большинство статистически значимых коэффициентов при независимой переменной InstColsV положительны. Представляется, что это можно трактовать как проявление взаимодополнения носителей различных уровней институционального коллективизма, приводящее к повышению четкости распределения ответственности в группе (см., напр.: [Griffith, Rubera, 2014]). В пользу этого предположения свидетельствует и то, что наибольшее количество статистически значимых коэффициентов наблюдается именно для зависимых переменных «Четкость делегирования задач...» и «Своевременность выполнения задач...», имеющих отношение к вопросам распределения ответственности в группе.

Дифференциал внутригруппового коллективизма (InGroupColsV). Среди статистически значимых коэффициентов независимой переменной In GroupColsV преобладают отрицательные (25 из 26).

В зависимых переменных «Успешность группы в генерации новых идей», «Четкость делегирования задач...» и «Качество анализа используемой информации» статистически значимые отрицательные коэффициенты есть в ответах на все три вопроса анкеты. Соответственно, можно вести речь о статистически значимой отрицательной взаимосвязи между ростом дифференциала InGroupColsV и воспринимаемой успешностью работы описываемых респондентами групп по данным критериям. Однако для кластера 4 данная взаимосвязь практически отсутствует. Так, в данном кластере статистически значимыми, причем с разным знаком, являются только два из трех коэффициентов зависимой переменной «Своевременность выполнения задач...». Предположительно, дифференциалы $S V$ каких-либо иных культурных измерений для данного кластера оказывают влияние, противоположное влиянию увеличения переменной InGroupColsV. Наиболее очевидно влияние переменных Inst ColSV (табл. 3).

Согласно [House et al., 2004, p.466-467], большинству культур присуща противоположная направленность институционального и внутригруппового кол- 
Диффференциал культурных измерений как метод анализа внутригрупповых процессов...

лективизма ввиду социально обусловленного различия интересов формальных общественных институтов и малых групп.

Вполне вероятно, что отрицательное влияние увеличения дифференциала InGroupColSV на воспринимаемую успешность работы кросс-культурных групп обусловлено склонностью носителей сравнительно низкого уровня ценностей внутригруппового коллективизма к оппортунистическому поведению (в частности, в форме фрирайдерства для студенческих команд) [Ralston et al., 2014].

В свою очередь, предполагаемое противонаправленное действие дифференциала InstColsV для кластера с наибольшим средним дифференциалом $S V$ может быть объяснено ориентацией носителей сравнительно высокого уровня институционального коллективизма на выполнение формальных правил и требований [Ching et al., 2014], что может быть более важно для работы группы, чем оппортунистический эффект увеличения дифференциала InGroupColsV.

Разделение культурного измерения коллективизма на институциональный и внутригрупповой появилось относительно недавно по сравнению с периодом теоретического осмысления и эмпирического обследования таких культурных измерений, как дистанция власти и избегание неопределенности. Несмотря на популярность и разработанность концепции культурного измерения коллективизма, полноценные эмпирические исследования соотношения институционального и внутригруппового коллективизма на уровне как национальной культуры в целом, так и малых групп в мировой и особенно российской литературе представлены недостаточно. Поэтому дальнейшие исследования в данной области являются одним из перспективных направлений развития теории и практики кросс-культурного менеджмента в российском и мировом контексте.

Результаты линейного регрессионного анализа для набора переменных $S V$ могут быть интерпретированы следующим образом.

1. Статистически значимые коэффициенты при независимых переменных $S V$ демонстрируют паттерны, логично интерпретируемые на основе результатов представленных в мировой литературе исследований в области анализа деятельности кросс-культурных команд, включая кросс-культурные студенческие группы. Исходя из этого и принимая во внимание ограниченность пилотного исследования с выборкой численностью в 84 человека, можно сделать вывод о том, что предлагаемый метод анализа дифференциалов культурных измерений, по крайней мере для дифференциалов $S V$, отражает реальные взаимосвязи культурных измерений и воспринимаемой участниками кросс-культурных групп успешности их деятельности. Однако, вероятно ввиду малого размера выборки данного пилотного исследования, в результатах анализа линейных регрессионных уравнений для дифференциалов SV статистически значимых коэффициентов в целом достаточно мало. Поэтому конкретные предположения, исходящие из анализа данных результатов, требуют проверки на более крупных выборках.

2. Для большинства независимых переменных дифференциалов SV характерно сравнительное преобладание положительных статистически значимых 
коэффициентов в кластерах 2 и 3 и более частое появление отрицательных в кластерах 1 и 4. Соответственно, для исследованной выборки увеличение каждого из дифференциалов $S V$ «при прочих равных» отрицательно влияет на воспринимаемую респондентами успешность работы кросс-культурных групп для культурно однородных групп (кластер 1) и групп с высоким уровнем культурного разнообразия (кластер 4). Для групп с умеренным уровнем культурного разнообразия (кластеры 2 и 3), напротив, это влияние является положительным.

3. Для независимой переменной In GroupColSV все статистически значимые коэффициенты, за исключением одного коэффициента в кластере 4, являются отрицательными. Таким образом, для исследованной выборки увеличение данного дифференциала отрицательно влияет на воспринимаемую респондентами успешность работы кросс-культурных групп по всем рассматриваемым критериям.

4. Для независимой переменной дифференциала GendEqSV отсутствуют статистически значимые коэффициенты в кластере 3; для независимой переменной дифференциала InstColsV нет статистически значимых коэффициентов в кластеpe 5. Можно предположить, что изменение данных дифференциалов для исследованной выборки статистически значимо влияет на воспринимаемую респондентами успешность работы для культурно однородных либо максимально культурно разнородных групп.

Рассмотрим результаты анализа независимых переменных $S P$ (табл. 4).

Аналогично порядку интерпретации представленных в табл. 3 результатов анализа независимых переменных $S V$ рассмотрим сначала общее предположение о взаимосвязи между дифференциалами $S P$ и воспринимаемым качеством работы кросс-культурных групп.

Можно предположить, что увеличение дифференциалов $S P$ приведет к синергии качеств, являющихся источником эффективности национальных деловых культур участников исследуемых групп. Однако в таком случае в результатах линейного регрессионного анализа переменных $S P$ появится количество статистически значимых коэффициентов с положительным знаком, аналогичное количеству коэффициентов с отрицательным знаком в результатах анализа переменных $S V$. В табл. 4 показан лишь один случай статистически значимых трех коэффициентов, а именно - для независимой переменной GendEqSP и зависимой переменной «Четкость делегирования задач...» в кластере 2. Соответственно, предположение о положительном влиянии увеличения дифференциалов $S P$ ввиду синергии положительных качеств национальных культур участников не подтверждается.

Приведем результаты линейного регрессионного анализа для отдельных независимых переменных.

Дифференциал дистанции власти (PDISP). Коэффициенты дифференциала PDISP ни в одном из кластеров и ни в одной из зависимых переменных не дают трех статистически значимых результатов для всех вопросов. Однако для данных коэффициентов можно отметить присутствие статистически значимых отрицательных коэффициентов в кластерах 1 и 4 и положительных коэффициен- 
Дифференциал культурных измерений как метод анализа внутригрупповых процессов...

Таблица 4. Результаты линейного регрессионного анализа для набора переменных $S P$

\begin{tabular}{|c|c|c|c|c|c|c|c|c|c|c|c|c|c|}
\hline \multirow[t]{2}{*}{ Переменные } & \multirow{2}{*}{$\bigvee_{\text {Кластер }}^{\text {Вопрос }}$} & \multicolumn{3}{|c|}{$\begin{array}{c}\text { Успешность } \\
\text { группы } \\
\text { в генерации } \\
\text { новых идей }\end{array}$} & \multicolumn{3}{|c|}{$\begin{array}{c}\text { Четкость } \\
\text { делегирования } \\
\text { задач в рамках } \\
\text { выполнения } \\
\text { проекта }\end{array}$} & \multicolumn{3}{|c|}{$\begin{array}{c}\text { Своевремен- } \\
\text { ность выполне- } \\
\text { ния задач в ходе } \\
\text { работы над } \\
\text { проектом }\end{array}$} & \multicolumn{3}{|c|}{$\begin{array}{c}\text { Качество } \\
\text { анализа } \\
\text { используемой } \\
\text { информации }\end{array}$} \\
\hline & & 51 & 52 & 53 & 61 & 62 & 63 & 71 & 72 & 73 & 81 & 82 & 83 \\
\hline \multirow[t]{4}{*}{ PDISP } & 1 & - & + & - & - & - & - & - & - & + & - & - & - \\
\hline & 2 & - & + & + & - & + & - & - & + & + & + & + & + \\
\hline & 3 & + & + & + & + & - & + & + & - & + & + & + & + \\
\hline & 4 & + & + & - & + & - & + & + & + & - & - & + & + \\
\hline \multirow{4}{*}{ UAISP } & 1 & - & - & - & - & + & - & - & + & - & - & - & + \\
\hline & 2 & + & + & - & - & + & + & - & - & - & - & + & + \\
\hline & 3 & + & + & + & + & + & + & - & + & + & + & + & + \\
\hline & 4 & + & + & + & + & + & + & + & + & - & - & - & + \\
\hline \multirow{4}{*}{ GendEqSP } & 1 & + & + & + & + & - & + & + & + & + & + & + & + \\
\hline & 2 & - & - & - & - & - & - & - & - & - & - & - & - \\
\hline & 3 & + & + & + & + & + & + & + & - & - & - & - & - \\
\hline & 4 & + & + & + & - & + & - & - & + & + & + & + & - \\
\hline \multirow{4}{*}{ InstColSP } & 1 & - & - & - & - & + & - & - & - & - & - & - & + \\
\hline & 2 & - & + & - & - & + & - & + & + & - & - & - & + \\
\hline & 3 & - & + & - & + & + & + & + & + & - & - & + & + \\
\hline & 4 & + & - & - & + & - & - & + & - & - & - & + & + \\
\hline \multirow{4}{*}{ In GroupColsP } & 1 & - & - & - & - & - & - & - & - & - & - & - & - \\
\hline & 2 & - & - & - & - & - & - & - & - & - & - & - & - \\
\hline & 3 & - & - & - & - & - & - & - & - & - & - & - & - \\
\hline & 4 & - & - & - & - & - & - & - & - & - & - & - & - \\
\hline
\end{tabular}

П р и ме ч а н и е: см. обозначения к табл. 3.

тов - в кластерах 3 и 4, в то время как в кластере 2 нет статистически значимых коэффициентов.

Соответственно можно сделать следующие предположения (безусловно, с необходимостью их проверки на большей по размеру выборке).

Во-первых, при незначительном уровне культурного разнообразия $S P$ в группе увеличение PDISP отрицательно влияет на воспринимаемую участниками успешность групп в четкости делегирования и своевременности выполнения задач, а также в качестве анализа используемой информации. Во-вторых, для групп с более высоким уровнем культурного разнообразия увеличение PDISP будет положительно влиять на воспринимаемую участниками успешность групп по всем исследуемым критериям, включая также и успешность групп в генерации новых идей.

Исходя из результатов исследований взаимосвязи дистанции власти и результативности деятельности кросс-культурных групп, описываемых в [Ferdman, 
Sagiv, 2012; Sheikh, Newman, Al Azzeh, 2013], в культурно однородных группах увеличение среднего уровня дистанции власти может ухудшать результативность группы по причине усиления в группе неконструктивных иерархических конфликтов. В то же время в более разнородных группах увеличение среднего уровня дистанции власти может приводить к улучшению координации деятельности участников и, таким образом, скорее повышать результативность группы [Sheikh, Newman, Al Azzeh, 2013].

Соответственно можно предположить, что в кластере 1 увеличение PDISP будет отрицательно влиять на деятельность группы из-за того, что увеличение этого дифференциала «при прочих равных» может создавать взаимное восприятие членов группы как склонных к иерархическим конфликтам и тем самым увеличивать потенциал возникновения неконструктивных конфликтов в группе. При расширении же культурного разнообразия группы в целом увеличение PDISP, вопервых, может в меньшей степени способствовать конфликтам, поскольку более привычные к культурному разнообразию члены группы могут и не воспринимать проявления увеличения этого дифференциала как конфликтное поведение. Вовторых, в группах с более высоким средним уровнем дифференциалов $S P$ увеличение PDISP «при прочих равных» может привести и к описанному в [Ferdman, Sagiv, 2012; Tsai et al., 2012] эффекту улучшения координации участников группы при увеличении разброса в ней значений параметра дистанции власти.

Дифференциал избегания неопределенности (UAISP). Несмотря на отсутствие случаев трех значимых коэффициентов для всех вопросов какой-либо из зависимых переменных, коэффициенты при независимой переменной UAISP характеризуются рядом особенностей.

Для данной переменной обращает на себя внимание преобладание отрицательных статистически значимых коэффициентов для кластеров 1 и 2 и положительных - для кластеров 3 и 4.

Подобное распределение знаков коэффициентов близко к ситуации, описанной для переменной PDISP. Аналогичным образом его можно интерпретировать как преимущественно негативное влияние увеличения UAISP на результаты деятельности культурно однородных групп и преимущественно позитивное влияние на результаты деятельности групп с высоким уровнем культурного разнообразия.

Согласно исследованиям [Ferdman, Sagiv, 2012; Tsai et al., 2012], можно высказать предположение, что увеличение разброса значений избегания неопределенности в группе может включать в себя как приводящие к развитию внутригрупповых конфликтов коммуникационные затруднения, так и улучшение четкости распределения ролей и компетенций, в целом повышающее результаты работы группы.

В культурно однородных группах увеличение UAISP может способствовать ухудшению коммуникации и возникновению конфликтов ввиду эффекта неожиданности возникающих поведенческих изменений при сохранении общей культурной однородности. Для групп с высоким уровнем культурного разнообразия, в свою очередь, увеличение UAISP способно улучшить координацию работы 
Дифференциал культурных измерений как метод анализа внутригрупповых процессов...

в группе ввиду более четкого распределения ролей, так как носители более низких уровней UAISP в таком случае будут более явно заметны и смогут быстрее занять место лидеров [Griffith, Rubera, 2014].

Дифференциал гендерного равенства (GendEqSP). Как отмечается в [House et al., 2004], сравнительно низкий уровень гендерного равенства связан с преимущественной ориентацией на достижение формального результата работы, а не на сохранение человеческих отношений (с чем в большей степени связан сравнительно высокий уровень данного культурного измерения).

Коэффициенты при независимой переменной GendEqSP, соответствующие трем вопросам одной зависимой переменной, статистически значимы для зависимой переменной четкости делегирования задач в кластере 2. Соответственно можно предположить, что для этого кластера увеличение разницы между сравнительной ориентацией участников группы на достижение результата и на сохранение отношений уменьшает степень взаимопонимания участников и поэтому затрудняет координацию выполняемых задач.

Аналогичное объяснение можно предложить и для статистически значимых отрицательных коэффициентов в отдельных вопросах зависимых переменных «Своевременность выполнения задач...» и «Качество анализа используемой информации», которые определяются качеством координации между участниками групп.

Для вопросов зависимой переменной «Успешность группы в генерации новых идей», наименее связанной с качеством внутригрупповой координации, все статистически значимые коэффициенты положительны. В вопросах, отражающих зависимые переменные «Четкость делегирования задач...», «Своевременность выполнения задач...» и «Качество анализа используемой информации», в различных кластерах также присутствуют и статистически значимые положительные коэффициенты, что подтверждает отсутствие значимого уровня проявления описанного выше отрицательного эффекта увеличения дифференциала GendEqSP.

Логично интерпретировать это как возможность конструктивного конфликта между носителями ориентации на достижение результата и ориентации на сохранение достижений (соотносящихся, соответственно, с высоким либо низким уровнем гендерного равенства $S P$ ). Однако эта интерпретация требует дополнительной проверки на более крупной выборке, так как результаты линейного регрессионного анализа показывают, что по крайней мере для одного из кластеров увеличение GendEqSP негативно влияет на четкость делегирования задач в рамках выполнения проекта, увеличивая, таким образом, вероятность возникновения конфликтов неконструктивного характера.

Дифференциал институционального коллективизма (InstColSP). Среди коэффициентов при независимой переменной InstColSP нет случаев трех значимых коэффициентов для всех вопросов какой-либо из зависимых переменных. Однако для большинства зависимых переменных (за исключением переменной «Качество анализа используемой информации») знаки статистически значимых 
коэффициентов соответствуют прослеживаемому и для независимых переменных PDISP и UAISP паттерну преобладания отрицательных коэффициентов для кластера 1 и положительных - для кластеров с более высоким средним уровнем дифференциалов $S P$.

Таким образом, в отношении большинства зависимых переменных можно предположить негативное влияние увеличения дифференциала InstColSP в культурно однородных и позитивное влияние - в культурно разнородных группах. Объяснением этих явлений могут быть эффекты неожиданности возникающих поведенческих изменений при сохранении общей культурной однородности «при прочих равных», в первом [Ferdman, Sagiv, 2012; Tsai et al., 2012] и улучшения координации в группе - во втором случае [Griffith, Rubera, 2014].

Особый интерес вызывает распределение знаков коэффициентов при независимой переменной InstColSP в уравнениях, описывающих зависимую переменную «Качество анализа исследуемой информации».

Здесь имеют место отрицательные статистически значимые коэффициенты в ответах на вопрос 8.1 (кластеры 1 и 4). Что касается вопроса 8.3, то в ответе на него имеют место положительные статистически значимые коэффициенты (кластеры 1 и 3). Согласно логике избыточности формулировок вопросов, эти вопросы повторяют друг друга. Однако их понимание респондентами все же не может не иметь незначительных смысловых расхождений; представляется, что последние при анализе более крупной выборки можно рассмотреть в качестве факторов, вызывающих разницу в коэффициентах.

Дифференциал внутригруппового коллективизма (InGroupColSP). Наконец, для независимой переменной In GroupColsP статистически значимыми стали отрицательные коэффициенты в кластерах 1 и 2 при отдельных вопросах всех зависимых переменных, за исключением переменной «Своевременность выполнения задач...».

Представляется, что отрицательное воздействие увеличения этой переменной на воспринимаемую респондентами успешность работы группы схоже с воздействием увеличения иных дифференциалов $S P$, за исключением GendEqSP. Так, для культурно однородных групп рост расхождений в таком значимом для взаимодействия студенческих команд с преподавателями и администрацией параметре, как внутригрупповой коллективизм, способен привести к возникновению конфликтных ситуаций прежде всего по поводу восприятия спорных вопросов этики взаимоотношения группы с данными контрагентами [Triandis et al., 1986; Rhee, Uleman, Lee, 1996; Realo, Allik, Vadi, 1997].

Однако при этом не очень понятно отсутствие как положительных, так и отрицательных статистически значимых коэффициентов в кластерах 3 и 4, т.е. в группах с относительно высоким уровнем общего культурного разнообразия. Вопрос об отсутствии подобных коэффициентов, безусловно, весьма интересен. Кроме этого, в целях дальнейших исследований необходимо изучение причины отсутствия в выборке статистически значимых влияний изменений In GroupColsP 
Диффференциал культурных измерений как метод анализа внутригрупповых процессов...

на зависимую переменную «Своевременность выполнения задач...». Для этой переменной логично предположить существование аналогичных взаимосвязей с независимой переменной InGroupColsP.

Итак, результаты линейного регрессионного анализа для набора переменных SP могут быть интерпретированы следующим образом.

1. Аналогично коэффициентам при независимых переменных дифференциалов SV, статистически значимые коэффициенты при независимых переменных дифференциалов $S P$ демонстрируют паттерны, которые можно логично интерпретировать, исходя из результатов исследований деятельности кросс-культурных студенческих групп и других кросс-культурных команд, представленных в зарубежных работах (см., напр.: [Bernardi, 2006; Ferdman, Sagiv, 2012; Ching et al., 2014; Griffith, Rubera, 2014; Ralston et al., 2014]). Таким образом (учитывая ограниченность выборки пилотного исследования), анализ дифференциалов культурных измерений SP также способен отражать реально существующие взаимосвязи и поэтому вполне может быть использован для анализа влияния культурных факторов на деятельность кросс-культурных команд.

2. Для всех независимых переменных наблюдается преимущественно отрицательное влияние увеличения дифференциалов $S P$ на воспринимаемую участниками успешность деятельности групп в кластерах 1 и 2, т. е. в достаточно культурно однородных группах. Это можно объяснить тем, что для культурно однородных групп увеличение дифференциала какого-либо из культурных измерений «при прочих равных» создает эффект неожиданности возникающих поведенческих изменений при сохранении общей культурной однородности. В результате допускается ухудшение коммуникации между участниками группы, способное привести к возникновению неконструктивных конфликтов.

3. Для всех независимых переменных, кроме InGroupColsP, в кластерах 3 и 4 характерно преимущественно положительное влияние увеличения дифференциалов на воспринимаемую участниками успешность деятельности групп. Объяснить это можно тем, что в группах с высоким уровнем культурного разнообразия повышение дифференциалов $S P$ может способствовать возникновению конструктивных конфликтов, а также взаимодополнению сильных сторон носителей различных уровней культурных измерений.

4. Для независимой переменной In GroupColSP статистически значимое влияние увеличения данного дифференциала на воспринимаемую участниками успешность деятельности групп наблюдается только в кластерах 1 и 2, а также зависимой переменной «Своевременность выполнения задач...». 


\section{ЗАКЛЮЧЕНИЕ}

Цель настоящего исследования - разработка и эмпирическая апробация специфического метода анализа внутригрупповых процессов в кросс-культурных командах. Для ее достижения был разработан метод, основанный на исследовании дифференциалов культурных измерений в этих командах.

Апробация указанного метода была проведена на эмпирическом материале кросс-культурных групп студентов, выполнявших групповые проектные работы в рамках обучения на ООП бакалавриата и магистратуры по направлениям «Менеджмент» и «Государственное и муниципальное управление»в Институте «Высшая школа менеджмента» СПбГУ. Исследование включало в себя анализ влияния дифференциалов ряда культурных измерений модели GLOBE (рассматриваемых и как нормативные ценности, и как социальные практики) на воспринимаемую участниками групп успешность их деятельности с точки зрения таких критериев, как успешность группы в генерации новых идей, четкость делегирования и своевременность выполнения задач в рамках работы над проектом, качество анализа используемой информации.

Статистически значимые результаты проведенного в ходе исследования линейного регрессионного анализа демонстрируют паттерны, логично интерпретируемые с учетом выводов имеющихся исследований в области анализа деятельности кросс-культурных команд, включая кросс-культурные студенческие группы. Таким образом, предлагаемый метод анализа дифференциалов культурных измерений отражает реальные взаимосвязи дифференциалов культурных измерений и воспринимаемой участниками кросс-культурных групп успешности их деятельности.

Преимущество разработанного метода состоит в его ориентированности на анализ процессов, происходящих внутри исследуемых команд. Это позволяет использовать метод для практических целей руководства деятельностью кросскультурных команд (по крайней мере, кросс-культурных групп студентов, выполняющих групповые проектные работы). В частности, выявленные различия в направленности влияния изменения дифференциалов культурных измерений для групп, отличающихся по уровню культурного разнообразия, можно учитывать при принятии решений о формировании состава групп (или подгрупп в рамках одной проектной группы) для выполнения различных типов проектов.

Важнейшее направление дальнейшего анализа касается проверки выявленных взаимосвязей на выборке более крупного размера. Безусловно, подобная выборка может иметь любую отраслевую принадлежность. Однако значимым представляется и продолжение анализа именно студенческих групп (необязательно интернациональных, так как различия в значениях культурных измерений наблюдаются и в однородных группах). Специфическим ограничением проведенного исследования выступает отсутствие в заполнявшейся респондентами анкете вопросов, касающихся численности участников групп. Исходя из этого, необ- 
Дифференциал культурных измерений как метод анализа внутригрупповых процессов...

ходимым видится сравнительный анализ исследуемых взаимосвязей в группах с разной численностью участников.

Что касается выявленных в настоящем исследовании связей между зависимыми и независимыми переменными, то с учетом результатов их анализа можно предложить следующие направления дальнейших исследований. Так, интерес представляет изучение соотношения и взаимодействия культурных измерений институционального и внутригруппового коллективизма. В частности, важно изучение влияния изменений дифференциалов внутригруппового коллективизма $S V$ и $S P$ на деятельность групп с высоким уровнем общего культурного разнообразия. Кроме того, актуален более подробный анализ полученных результатов положительного влияния увеличения дифференциала гендерного равенства (как нормативной ценности, так и социальной практики) на воспринимаемую участниками групп успешность их деятельности в связи с тем, что они противоречат существующим теоретическим концепциям и эмпирическим выводам в области кросс-культурного менеджмента.

\section{Литература}

Солдатова Г. У. Психология межэтнической напряженности. М.: Смысл, 1998.

О внесении изменений в федеральные государственные образовательные стандарты высшего профессионального образования. Приказ Министра образования и науки Российской Федерации от 20 мая 2010 г. № 544.

Abbondante P., Caple S., Ghazzawi I., Schantz G. Learning Communities and Experiential Entrepreneurial Success // Academy of Educational Leadership Journal. 2014. Vol. 18, N 2. P. 13-34.

Bernardi R. Associations between Hofstede's Cultural Constructs and Social Desirability Response Bias // Journal of Business Ethics. 2006. Vol. 65, N 1. P. 43-53.

Besteiro É. N.C., de Souza Pinto J., Novaski O. Success Factors in Project Management // Business Management Dynamics. 2015. Vol. 4 (March), N 9. P. 19-34.

Ching G. V., Hoffman J.J., Qing C., Schniederjans M. J. The Effects of Organizational Culture on IT Project Performance: A Moderation Perspective // International Journal of Project Management. 2014. Vol. 32, N 7. P. 1170-1181.

Connaughton S. L., Shuffler M. Multinational and Multicultural Distributed Teams: A Review and Future Agenda // Small Group Research. 2007. Vol.38, N 3. P. 387-412.

Ferdman B.M., Sagiv L. Diversity in Organizations and Cross-Cultural Work Psychology: What If They Were More Connected? // Industrial and Organizational Psychology. 2012. Vol.5, N 3. P. 323-345.

Gonzalez-Padron T., Ferguson J. M. Using a Service Audit Project for Improving Student Learning in a Service-Marketing Course // Marketing Education Review. 2015. Vol.25, N 2, P.99-116.

Griffith D. A., Rubera G. A Cross-Cultural Investigation of New Product Strategies for Technological and Design Innovations // Journal of International Marketing. 2014. Vol. 22, N 1. P.5-20,

Hall E. T. The Silent Language. New York: Anchor, 1959.

Hall T. W., Higson A. W., Pierce B. J., Price K. H., Skousen C. J. Haphazard Sampling: Selection Biases Induced by Control Listing Properties and the Estimation Consequences of these Biases // Behavioral Research in Accounting. 2012. Vol.24, N 2. P. 101-132.

Hofstede G. Motivation, Leadership, and Organization: Do American Theories Apply Abroad? // Organizational Dynamics. 1980. Vol.9, N 1. P.42-63. 
House R.J., Hanges P.J., Javidan M., Dorfman P.W., Gupta V.(eds.). Culture, Leadership, and Organizations: The GLOBE Study of 62 Societies. Thousand Oaks, CA, London, New Delhi: Sage Publications, Inc., 2004.

Knights D., Tullberg M. Managing Masculinity/Mismanaging the Corporation // Organization. 2012. Vol. 19, N 4. P.385-404.

Lehmann C. M., Heagy C. D., Wilson V. A Realistic Test of Transfer: Do Novices in Established Groups Represent Problems Similarly to Experienced Information Systems Professionals after Group Interaction? // Issues in Accounting Education. 2011. Vol. 26, issue 1 (February). P. 39-65.

Mansour H. F. Enhancing First Year Management Students' Engagement: An Action Research Project to Explore the Use of the Essay Feedback Checklist (EFC) // International Journal of Management Education (Elsevier Science). 2015. Vol. 13, issue 3 (November). P. 218-226.

Osgood C.E. The Nature and Measurement of Meaning // Psychological Bulletin. 1952. Vol.49, N 3. P. 197-237.

Ralston D., Egri C., Furrer O., Kuo M.-H., Li Y., Wangenheim F., Dabic M., Naoumova I., Shimizu K., Garza Carranza M., Fu P., Potocan V., Pekerti A., Lenartowicz T., Srinisavan N., Casado T., Rossi A., Szabo E., Butt A., Palmer I. Societal-Level versus Individual-Level Predictions of Ethical Behavior: A 48-Society Study of Collectivism and Individualism // Journal of Business Ethics. 2014. Vol.122, N 2. P. 283-306.

Realo A., Allik J., Vadi M. The Hierarchical Structure of Collectivism // Journal of Research in Personality. 1997. Vol.31, N 1. P.93-116.

Rhee E., Uleman J. S., Lee H. K. Variations in Collectivism and Individualism by In-group and Culture: Confirmatory Factor Analyses // Journal of Personality and Social Psychology. 1996. Vol. 71, N 5. P. 1037-1054.

Samkin G., Francis G. Introducing a Learning Portfolio in an Undergraduate Financial Accounting Course // Accounting Education. 2008, Vol. 17, ISSUE 3 (September). P. 233-271.

Sheikh A.Z., Newman A., Al Azzeh S. A.-F. Transformational Leadership and Job Involvement in the Middle East: the Moderating Role of Individually Held Cultural Values // The International Journal of Human Resource Management. 2013. Vol.24, N 6. P. 1077-1095.

Singh B. J., Joshi R. Benchmarking Academics through Sustainable Assessment Criteria: An Indian Case Study // Benchmarking: An International Journal. 2015. Vol. 22, N 3. P. 505-536.

Sormunen E., Tanni M., Alamettälä T., Heinström J. Students' Group Work Strategies in Source-Based Writing Assignments // Journal of the Association for Information Science \& Technology. 2014. Vol. 65, ISSUE 6 (June). P. 1217-1231.

Spony G. The Development of a Work-Value Model Assessing the Cumulative Impact of Individual and Cultural Differences on Managers' Work-Value Systems: Empirical Evidence from French and British Managers // The International Journal of Human Resource Management. 2003. Vol. 14, N 4. P. 658-679.

Triandis H.C., Bontempo R., Bentancourt H., Bond M., Leung K., Brenes A., et al. The Measurement of the Etic Aspects of Individualism and Collectivism across Cultures // Australian Journal of Psychology. 1986. Vol.38, N 3. P.257-267.

Tsai W.C., Chi N.W., Grandey A.A., Fung S. C. Exploring Boundary Conditions of the Relationship between Positive Group Affective Tone and Team Creativity: Negative Group Affective Tone and Team Trust as Moderators // Journal of Organizational Behavior. 2012. Vol.33, N 5. P. 638-656.

Trompenaars F. Riding the Waves of Culture: Understanding Diversity in Global Business. Burr Ridge, IL: Irwin Professional Pub., 1994.

Для цитирования: Благов Е. Ю., Гиленко Е. В., Башлыкова А.С. Дифференциал культурных измерений как метод анализа внутригрупповых процессов в кросс-культурных командах // Вестн. С.-Петерб. ун-та. Сер. 8. Менеджмент. 2016. Вып. 2. C. 98-126. DOI: 10.21638/11701/ spbu08.2016.205 
Дифференциал культурных измерений как метод анализа внутригрупповых процессов...

\section{References}

Soldatova G. U. Psikhologiia mezhetnicheskoi napriazhennosti [Psychology of Interethnic Tension]. Moscow, Smysl Publ., 1998.

$\mathrm{O}$ vnesenii izmenenii $\mathrm{v}$ federal'nye gosudarstvennye obrazovatel'nye standarty vysshego professional'nogo obrazovaniia [On Amendments to the Federal State Educational Standards of Higher Professional Education]. Prikaz Ministra obrazovaniia i nauki Rossiiskoi Federatsii [Order of the Minister of Education and Science of the Russian Federation]. May 20, 2010. N 544. (In Russian)

Abbondante P., Caple S., Ghazzawi I., Schantz G. Learning Communities and Experiential Entrepreneurial Success. Academy of Educational Leadership Journal, 2014, vol. 18, no. 2, pp. 13-34.

Bernardi R. Associations between Hofstede's Cultural Constructs and Social Desirability Response Bias. Journal of Business Ethics, 2006, vol. 65, no. 1, pp. 43-53.

Besteiro É. N. C., de Souza Pinto J., Novaski O. Success Factors in Project Management. Business Management Dynamics, 2015, vol. 4, no. 9 (March), pp. 19-34.

Ching G. V., Hoffman J. J., Qing C., Schniederjans M. J. The Effects of Organizational Culture on IT Project Performance: A Moderation Perspective. International Journal of Project Management, 2014, vol. 32, no. 7, pp. 1170-1181.

Connaughton S.L., Shuffler M. Multinational and Multicultural Distributed Teams: A Review and Future Agenda. Small Group Research, 2007, vol. 38, no. 3, pp. 387-412.

Ferdman B. M., Sagiv L. Diversity in Organizations and Cross-Cultural Work Psychology: What If They Were More Connected? Industrial and Organizational Psychology, 2012, vol. 5, no. 3, pp. 323-345.

Gonzalez-Padron T., Ferguson J. M. Using a Service Audit Project for Improving Student Learning in a Service-Marketing Course. Marketing Education Review, 2015, vol. 25, no. 2, pp. 99-116.

Griffith D. A., Rubera G. A Cross-Cultural Investigation of New Product Strategies for Technological and Design Innovations. Journal of International Marketing, 2014, vol. 22, no. 1, pp. 5-20,

Hall E. T. The Silent Language. New York, Anchor Publ., 1959.

Hall T. W., Higson A. W., Pierce B. J., Price K. H., Skousen C. J. Haphazard Sampling: Selection Biases Induced by Control Listing Properties and the Estimation Consequences of these Biases. Behavioral Research in Accounting, 2012, vol. 24, no. 2, pp. 101-132.

Hofstede G. Motivation, Leadership, and Organization: Do American Theories Apply Abroad? Organizational Dynamics, 1980, vol. 9, no. 1, pp. 42-63.

Culture, Leadership, and Organizations: The GLOBE Study of 62 Societies. Eds R. J. House, P. J. Hanges, M. Javidan, P.W. Dorfman, V. Gupta. Thousand Oaks, CA, London, New Delhi, Sage Publications, Inc., 2004.

Knights D., Tullberg M. Managing Masculinity/Mismanaging the Corporation. Organization, 2012, vol. 19 , no. 4, pp. 385-404.

Lehmann C.M., Heagy C.D., Wilson V.A Realistic Test of Transfer: Do Novices in Established Groups Represent Problems Similarly to Experienced Information Systems Professionals after Group Interaction? Issues in Accounting Education, 2011, vol. 26, issue 1 (February), pp. 39-65.

Mansour H. F. Enhancing First Year Management Students' Engagement: An Action Research Project to Explore the Use of the Essay Feedback Checklist (EFC). International Journal of Management Education (Elsevier Science), 2015, vol. 13, issue 3 (November), pp. 218-226.

Osgood C.E. The Nature and Measurement of Meaning. Psychological Bulletin, 1952, vol. 49, no. 3, pp. 197-237.

Ralston D., Egri C., Furrer O., Kuo M.-H., Li Y., Wangenheim F., Dabic M., Naoumova I., Shimizu K., Garza Carranza M., Fu P., Potocan V., Pekerti A., Lenartowicz T., Srinisavan N., Casado T., Rossi A., Szabo E., Butt A., Palmer I. Societal-Level versus Individual-Level Predictions of Ethical Be- 
havior: A 48-Society Study of Collectivism and Individualism. Journal of Business Ethics, 2014, vol. 122 , no. 2, pp. 283-306.

Realo A., Allik J., Vadi M. The Hierarchical Structure of Collectivism. Journal of Research in Personality, 1997, vol. 31, no. 1, pp. 93-116.

Rhee E., Uleman J. S., Lee H. K. Variations in Collectivism and Individualism by In-group and Culture: Confirmatory Factor Analyses. Journal of Personality and Social Psychology, 1996, vol. 71, no. 5, pp. 1037-1054.

Samkin G., Francis G. Introducing a Learning Portfolio in an Undergraduate Financial Accounting Course. Accounting Education, 2008, vol. 17, issue 3 (September), pp. 233-271.

Sheikh A.Z., Newman A., Al Azzeh S. A.-F. Transformational Leadership and Job Involvement in the Middle East: the Moderating Role of Individually Held Cultural Values. The International Journal of Human Resource Management, 2013, vol. 24, no. 6, pp. 1077-1095.

Singh B. J., Joshi R. Benchmarking Academics through Sustainable Assessment Criteria: An Indian Case Study. Benchmarking: An International Journal, 2015, vol. 22, no. 3, pp. 505-536.

Sormunen E., Tanni M., Alamettälä T., Heinström J. Students' Group Work Strategies in Source-Based Writing Assignments. Journal of the Association for Information Science \& Technology, 2014, vol. 65 , issue 6 (June), pp. 1217-1231.

Spony G. The Development of a Work-Value Model Assessing the Cumulative Impact of Individual and Cultural Differences on Managers' Work-Value Systems: Empirical Evidence from French and British Managers. The International Journal of Human Resource Management, 2003, vol. 14, no. 4, pp. 658-679.

Triandis H.C., Bontempo R., Bentancourt H., Bond M., Leung K., Brenes A., et al. The Measurement of the Etic Aspects of Individualism and Collectivism across Cultures. Australian Journal of Psychology, 1986, vol. 38, no. 3, pp. 257-267.

Tsai W.C., Chi N. W., Grandey A. A., Fung S. C. Exploring Boundary Conditions of the Relationship between Positive Group Affective Tone and Team Creativity: Negative Group Affective Tone and Team Trust as Moderators. Journal of Organizational Behavior, 2012, vol. 33, no. 5, pp. 638-656.

Trompenaars F. Riding the Waves of Culture: Understanding Diversity in Global Business. Burr Ridge, IL: Irwin Professional Publ., 1994.

For citation: Blagov E. Yu., Gilenko E. V., Bashlykova A.S. Cross-Cultural Dimensions Differential as a Method for Analyzing In-Group Processes in Cross-Cultural Teams. Vestnik of Saint-Petersburg University. Ser. 8. Management, 2016, issue 2, pp. 98-126. DOI: 10.21638/11701/spbu08.2016.205

Статья поступила в редакцию 31 декабря 2015 г.; принята к печати 20 апреля 2016 г.

Контактная информация

Благов Евгений Юрвевич - кандидат экономических наук, ассистент; blagove@gsom.pu.ru

Гиленко Евгений Валерьевич - кандидат экономических наук, доцент;

e.gilenko@gsom.pu.ru

Башлыкова Ангелина Сергеевна - студент магистратуры;

Bashlikova.bm2015@ledu.gsom.pu.ru

Blagov Evgeniy Yu. - Candidate of Economic Sciences, Assistant Professor;

blagove@gsom.pu.ru

Gilenko Evgenii V. - Candidate of of Economic Sciences, Associate Professor;

e.gilenko@gsom.pu.ru

Bashlykova Angelina S. — Master Program Student; Bashlikova.bm2015@ledu.gsom.pu.ru 